Background trends were estimated using a weighted mean of control countries' rates.

Results Although pregnancies and births in England fell during the Strategy period, we found no difference compared with control. In interrupted time series analyses, trends in rates of teenage pregnancy beginning in 1999 in England were similar to Scotland $(0.08$ fewer pregnancies per 1,000 women per year; -0.74 to 0.60$)$ and Wales $(0.14$ more pregnancies per 1,000 women per year; -0.48 to 0.76 ). In synthetic control analyses, yearly under-18 birth rates in England were very similar to synthetic control predictions post intervention, and under-20 pregnancy rates were marginally higher than control across the post intervention time period. Placebo testing and other sensitivity analyses supported the finding of no effect.

Conclusion Our analyses cast doubt on the effectiveness of England's teenage pregnancy strategy. These results should be factored into decision making if other countries, or England in the future, were to contemplate similar costly strategies.

\section{OP92 DOES MATERNAL FOLIC ACID SUPPLEMENTS IN PREGNANCY INFLUENCE AUTISM SPECTRUM DISORDER IN CHILDREN? A SYSTEMATIC REVIEW AND META- ANALYSIS}

${ }^{1} \mathrm{C}$ Friel ${ }^{*},{ }^{1} \mathrm{AH}$ Leyland, ${ }^{2} \mathrm{~J}$ Anderson, ${ }^{1} \mathrm{M}$ Shimonovich, ${ }^{1} \mathrm{R}$ Dundas. ${ }^{1} \mathrm{MRC} / \mathrm{CSO}$ Social and Public Health Sciences Unit, University of Glasgow, Glasgow, UK; ${ }^{2}$ Public Health Research Group, University of Glasgow, Glasgow, UK

\subsection{6/jech-2020-SSMabstracts.90}

Background Folic acid supplements during pregnancy may be causally associated with reduced risk of Autism Spectrum Disorder (ASD). This relationship is potentially socially patterned as folic acid intakes are lower in deprived groups. The main limitations of previous systematic reviews and meta-analysis are multiple counts of the same unit of analysis and use of DerSimonian and Laird estimator which underestimates uncertainty and increases type I error rate. We aimed to evaluate evidence of a causal association between prenatal folic acid intake and offspring ASD, and outline evidence of health inequalities.

Methods

Results A total of 1,001,424 children (4646 cases) were pooled from five cohort and four case-control studies. No association was observed between maternal folic acid supplement intakes and offspring ASD (RR 0.77, 95\% CI: 0.53 $\left.1.11, \mathrm{I}^{2}=92.8 \%\right)$. However, when restricting to high quality studies, there was some evidence of a reduced risk of ASD (RR $0.76,95 \%$ CI: $0.56-1.03, \mathrm{I}^{2}=77 \%$ ) or with removal of the outlier (RR 0.69, 95\% CI: 0.51-0.94, $\mathrm{I}^{2}=93.2 \%$ ). A study was considered an outlier if the studies 95\% CI did not overlap the $95 \%$ CI of the summary effect estimate. Evidence of health inequalities was not reported in any study, but all considered socioeconomic position or an indicator of socioeconomic position to be an important confounder.

Conclusion High quality studies demonstrated a reduced risk of ASD in relation to folic acid supplements in pregnancy, yet residual confounding was likely. Nonconventional approaches to estimate causality were applied in only a few studies but they suggest the relationship is potentially causal. Future research should apply causally informed methods and investigate evidence of health inequalities.

\section{OP93 \\ THE ASSOCIATION BETWEEN GESTATIONAL AGE AT BIRTH AND SPECIAL EDUCATION NEEDS AT AGE 11 - EVIDENCE FROM THE UK MILLENNIUM COHORT STUDY}

${ }^{1} \mathrm{~N}$ Alterman*, ${ }^{2} \mathrm{~S}$ Johnson, ${ }^{1} \mathrm{C}$ Carson, ${ }^{3} \mathrm{~S}$ Petrou, ${ }^{1} \mathrm{O}$ Rivero-Arias, ${ }^{1} \mathrm{JJ}$ Kurinzcuk, ${ }^{4} \mathrm{AJ}$ Macfarlane, ${ }^{2} \mathrm{E}$ Boyle, ${ }^{1} \mathrm{MA}$ Quigley. ${ }^{1}$ Nuffield Department of Population Health, University of Oxford, Oxford, UK; ${ }^{2}$ Department of Health Sciences, University of Leicester, Leicester, UK; ${ }^{3}$ Nuffield Department of Primary Care Health Sciences, University of Oxford, Oxford, UK; ${ }^{4}$ Department of Health Sciences, City University London, London, UK

\subsection{6/jech-2020-SSMabstracts.91}

Background Being born at an earlier gestational age is a risk factor for having neurodevelopmental impairment and special educational needs (SEN) at school. Most prior studies focused on children born preterm ( $<37$ weeks) rather than across the entire gestational age continuum and have not examined specific types of SEN. Our aim was to examine the association between gestational age across the entire gestation spectrum and SEN at the age of 11 years.

Methods We analysed parent reported survey data from the UK Millennium Cohort Study, a nationally representative cohort of children born during the years 2000-2002. Information about the child's birth, health and sociodemographic factors was collected from mothers when children were 9 months old. Information about presence of SEN, whether the child has a statement of SEN which indicates more complex needs, and the reasons for SEN was collected from parents at age 11. Adjusted risk ratios (aRR) were estimated using modified Poisson regression while accounting for confounding.

Results The sample included 12,081 children with data at both time points. The proportion of children with SEN in the cohort was $11.2 \%$ and this proportion increased markedly as gestation decreased. The risk of having SEN was highest in children born before 32 weeks gestation who had a 3-fold higher risk than those born at 40 weeks $(\mathrm{aRR}=2.89$; 95\% CI $2.02,4.13)$. The risk was also elevated in children born early term (37-38 weeks) $(\mathrm{aRR}=1.33$; 95\% CI 1.11, 1.59). The proportion of children with a statement of SEN was $4.9 \%$ in the overall cohort. There was a clear inverse association between gestational age and having a statement of SEN, which peaked at $15.3 \%$ in children born $<32$ weeks $(a R R=3.96$; $95 \%$ CI $2.24,7.06)$. Gestational age was also inversely associated with having multiple reasons for SEN, reaching $\mathrm{aRR}=3.32(95 \%$ CI 1.61, 6.84) at $<32$ weeks.

Conclusion Children born at earlier gestational age are more likely to experience SEN, have more complex SEN and require support in multiple facets of learning at age 11 . This increased likelihood of SEN was observed even among children born at early term gestation.

\section{OP94 I I NEEDED SOMEBODY LIKE HER IN MY LIFE': MIGRANT WOMEN'S EXPERIENCES AND PERCEPTIONS OF HEALTH NAVIGATION SERVICES TO INCREASE ACCESS TO MATERNITY CARE IN THE UK}

${ }^{1} \mathrm{CA}$ Braham*, 'E Such, ${ }^{2} \mathrm{~S}$ Salway. ${ }^{1}$ School of Health and Related Research, University of Sheffield, Sheffield, UK; ${ }^{2}$ Department of Sociological Studies, University of Sheffield, Sheffield, UK

\subsection{6/jech-2020-SSMabstracts.92}

Background Migrant women in the UK are at increased risk of maternal morbidity and mortality compared to UK-born women, in part due to inequalities in healthcare access. Health 